\title{
Iguratimod, a Disease-Modifying Anti-Rheumatic Drug, Inhibits Osteoclastogenesis and Bone Resorption through Suppression of the Nuclear Factor of Activated T Cells Signaling Pathway
}

\author{
Jun Shiota, Hidetoshi Murao, Akihiko Miura, Masaaki Mikami, Keiichi Tanaka* \\ Research Laboratories, Toyama Chemical Co., Ltd., Toyama, Japan \\ Email: *keiichi.a.tanaka@fujifilm.com
}

How to cite this paper: Shiota, J., Murao, H., Miura, A., Mikami, M. and Tanaka, K. (2016) Iguratimod, a Disease-Modifying Anti-Rheumatic Drug, Inhibits Osteoclastogenesis and Bone Resorption through Suppression of the Nuclear Factor of Activated $\mathrm{T}$ Cells Signaling Pathway. Open Journal of Rheumatology and Autoimmune Diseases, 6, 106-119.

http://dx.doi.org/10.4236/ojra.2016.64017

Received: October 17, 2016

Accepted: November 18, 2016

Published: November 21, 2016

Copyright $\odot 2016$ by authors and Scientific Research Publishing Inc. This work is licensed under the Creative Commons Attribution International License (CC BY 4.0).

http://creativecommons.org/licenses/by/4.0/ (c) (i) Open Access

\begin{abstract}
Introduction: The aim of this study was to observe an inhibition of bone resorption and osteoclastogenesis by iguratimod (IGU, T-614), a disease-modifying anti-rheumatic drug, using adjuvant-induced arthritis (AIA) rats and receptor activator of nuclear factor kappa-B ligand (RANKL)-stimulated RAW264.7 cells. Methods: The bone mineral density and 3D morphometric parameters of hind paws in AIA rats were measured using micro computed tomography $(\mu \mathrm{CT})$ imaging. The activity of osteoclast cells was estimated based on tartrate-resistant acid phosphatase (TRAP) staining in specimens from the rats. In vitro TRAP activity was investigated using RANKL-stimulated RAW264.7 cells. The amount of nuclear factor of activated Tcells, cytoplasmic, calcineurin-dependent 1 (NFATc1) protein was measured by western blot analysis. The expression of $N$ fatcl, its regulator genes, its upstream factors, and osteoclast-functional genes were investigated. Results: In addition to the suppression of bone resorption and lesions of bone trabeculae of AIA rats, IGU significantly decreased the number of TRAP-positive cells in the calcaneal bones. Moreover, this drug inhibited the differentiation of RANKL-stimulated RAW264.7 cells into osteoclasts, which were identified morphologically and functionally. IGU decreased the amount of NFATc1 protein and improved the altered expression of NFATc1-associated genes and osteoclast-functional genes. Conclusions: IGU suppressed osteoclastogenesis and bone resorption via the RANKL-NFATc1 pathway, suggesting such effect would be expected in clinical use.
\end{abstract}

\section{Keywords}

Bone Resorption, Iguratimod, NFATc1, Osteoclast, Rheumatoid Arthritis 


\section{Introduction}

Iguratimod (IGU, T-614) is currently prescribed as a disease-modifying anti-rheumatic drug (DMARD) in Japan [1]. Good efficacy and safety were reported for IGU treatment in clinical trials and post-marketing surveillance in Japanese patients with rheumatoid arthritis (RA) [2] [3] [4].

IGU inhibits the production of inflammatory cytokines in cultured monocyte THP-1 cells and synovial cells from patients with RA through the suppression of nuclear factor kappa-B (NF- $\kappa \mathrm{B}$ ) activation [5]. It also inhibits the immunoglobulin production by direct action on $B$ cells without cytostatic effect [6]. Furthermore, IGU exhibits anti-inflammatory effects and improves abnormal immunological conditions in various animal models of inflammation and autoimmune diseases, including RA [7], [8]. In particular, IGU suppresses bone destruction in collagen-induced arthritis (CIA) mice [9], [10] and rats [11]. Recently, Gan et al. [12] have reported that IGU suppresses that receptor activator of NF- $\mathrm{KB}$ ligand (RANKL)-induced osteoclast differentiation and migration in RAW264.7 cells via NF- $\mathrm{KB}$ and mitogen-activated protein kinase (MAPK) pathways. This report is particularly interesting in connection with its stimulating action on osteoblastic differentiation that was shown by Kuriyama et al. [13].

On the other hand, it was reported that a nuclear factor of activated T cells (NFAT) signaling pathway was involved in the osteoclast-derived bone destruction [14] [15] [16]. Therefore, we investigated in vivo effect of IGU on bone destruction and resorption in adjuvant-induced arthritis (AIA) model rats. In addition, the in vitro effect on NFAT-signaling pathway in RANKL-stimulated RAW264.7 cells was examined in order to elucidate the detailed mechanism underlying the suppressive effect of IGU on bone destruction.

\section{Materials and Methods}

\subsection{Materials}

Iguratimod (IGU) was synthesized in Toyama Chemical Co., Ltd. (Tokyo, Japan). Other materials were obtained from the following sources: Mycobacterium tuberculosis H37 Ra (BD Diagnostic Systems, Hunt Valley, MD, USA), Dulbecco's modified Eagle medium (DMEM) (Nissui Pharmaceutical, Tokyo, Japan), fetal bovine serum (FBS) (Hyclone, Logan, UT, USA), non-essential amino acid (NEAA) (Invitrogen, Carlsbad, CA, USA), kanamycin (Sigma-Aldrich, St. Louis, MO, USA), RANKL (R \& D Systems, Minneapolis, MN, USA, and Peprotech, Rocky Hill, NJ, USA), bone resorption assay kit 48 (PG Research, Tokyo, Japan), mouse anti-NFATc1 monoclonal antibody (mAb) (7A6) (Santa Cruz Biotechnology, Santa Cruz, CA, USA), nuclear extract kit (Active Motif, Carlsbad, CA, USA), ECL Plus (GE Healthcare Bio-Sciences, Piscataway, NJ, USA), ReliaPrep RNA Cell Miniprep System (Promega, Madison, WI, USA), PrimeScript reverse transcriptase (Takara Bio Inc, Shiga, Japan), and SYBR Premix Ex Taq (Takara Bio).

\subsection{Animals and Cells}

The animal study was conducted in accordance with the laboratory animal use man- 
agement regulation at Toyama Chemical. Male Lewis rats were purchased from Charles River Japan (Kanagawa, Japan). The animals were housed in an air-conditioned room settled at $24^{\circ} \mathrm{C} \pm 2{ }^{\circ} \mathrm{C}$ and $50 \% \pm 20 \%$ of relative humidity, under a $12 \mathrm{~h}$ light-dark cycle, and fed with a commercial diet (CRF-1, Oriental Yeast; Tokyo, Japan) and received tap water ad libitum. A mouse macrophage cell line RAW264.7 cells (ATCC No. TIB-71) was purchased from Dainippon Pharmaceutical (Osaka, Japan). The cells were maintained in DMEM supplemented with $10 \% \mathrm{FBS}, 6 \mu \mathrm{g} / \mathrm{ml}$ of kanamycin and NEAA.

\subsection{Adjuvant-Induced Arthritis (AIA)}

Arthritis was induced by $M$. tuberculosis injection to the left hind paw of rats on day 0 using previously described methods [17] with modifications. On day 0 , the volumes of both hind paws were measured with a plethysmometer (Ugo Basile, Comerio VA, Italy) and animals were randomly divided into three groups as follows: (1) untreated and vehicle-administered group (normal), (2) AIA-induced and vehicle-administered group (control), (3) AIA-induced and IGU-administered group (10 mg/kg/day). IGU suspension was prepared in a solution of $0.5 \%$ methylcellulose 400 and administered orally once daily.

On day 21, the volumes of both hind paws were measured. The swelling rate of each paw was calculated by using the following formula: Swelling rate $(\%)=($ foot volume on day 21 -foot volume on day 0 )/foot volume on day $0 \times 100$.

\subsection{Radiography and Micro Computed Tomography ( $\mu \mathrm{CT})$ Imaging}

On day 21 after AIA induction, both hind paws were collected after euthanasia and fixed in 10\% formalin buffer (pH7.4). Digital radiography of both hind paws was pictured using $\mu \mathrm{FX}-1000$ and BAS-5000 systems (Fujifilm, Tokyo, Japan). Images of each calcaneum were taken using cone beam $\mu$ CT (MCT-100CB, Hitachi Medical, Chiba, Japan) with $25 \mu \mathrm{m}$ isotropic voxel resolution. The images of 3D microstructure were reconstituted using TRI/3D-BON (Ratoc System Engineering, Tokyo, Japan). The bone mineral density (BMD), 3D morphometric parameters [Bone volume/Tissue volume (BV/TV), trabecular bone pattern factor (TBPf), and structural model index (SMI)] were calculated [18].

\subsection{Histological Staining of Hind Paws}

Both hind paws were fixed in 10\% paraformaldehyde, decalcified in 10\% EDTA for one month at $4^{\circ} \mathrm{C}$, and embedded in paraffin. For histological analysis, $4-\mu \mathrm{m}$ sections were stained using hematoxylin-eosin or tartrate-resistant acid phosphatase/alkaline phosphatase (TRAP/ALP) stain kit (Wako pure chemical, Osaka, Japan). For TRAP staining, each paraffin section was dewaxed and rehydrated. TRAP staining was performed according to the manufacturer's instructions and nuclei were stained with methyl green. All TRAP-positive cells in the calcaneum on each section were counted under a microscope. 


\subsection{TRAP Activity and TRAP Staining in RANKL-Stimulated RAW264.7 Cells}

TRAP activity was measured as described by Hirotani et al. [19] with modifications. Briefly, RAW264.7 cells were cultured in a 96-well plate at a density of $1 \times 10^{4}$ cells/well and stimulated with $50 \mathrm{ng} / \mathrm{ml}$ of RANKL in the absence or presence of IGU. Six days after stimulation, the cells were harvested and TRAP activity was measured using the TRACP \& ALP assay kit (Takara Bio). For TRAP staining, the cells were fixed and stained using the TRAP staining kit (Primary Cell, Hokkaido, Japan). All TRAP-positive multinucleated ( $>3$ nuclei) cells (MNCs) were counted in each well.

\subsection{Bone Resorption in RANKL-Stimulated RAW264.7 Cells}

Bone resorption activity was assessed in a fluoresceinated calcium phosphate-coated plate provided with the bone resorption assay kit. Briefly, RAW264.7 cells were stimulated with $50 \mathrm{ng} / \mathrm{ml}$ of RANKL. Six days after stimulation, $100 \mu \mathrm{l}$ of the culture supernatant was transferred to a new 96-well plate and mixed with $50 \mu$ of bone resorption assay buffer. The fluorescence intensity was measured using a plate reader (Infinite M-200pro, Tecan, Männedorf, Switzerland) with excitation and emission wavelength of 485 and $535 \mathrm{~nm}$, respectively. On day 9 , the coated plate was washed with $5 \%$ sodium hypochlorite and pictures of each well were acquired under a microscope.

\subsection{Protein Preparation and Western Blot Analysis}

Twenty-four hours after RANKL stimulation ( $50 \mathrm{ng} / \mathrm{ml}$ ), the nuclear and cytoplasmic extracts from RAW264.7 cells were prepared using a nuclear extract kit. The samples (10 $\mu$ g protein/lane) were separated by $10 \%-20 \%$ gradient SDS-PAGE and transferred to polyvinylidene fluoride membrane (Merck Millipore, Billerica, MA, USA). After blocking with $5 \%$ skim milk in Tris buffered saline (TBS) containing 0.05\% Tween 20 for $1 \mathrm{~h}$, the membrane was incubated overnight with a mouse monoclonal antibody against NFATc1. After washing the membrane with TBS, the membrane was incubated for $1 \mathrm{~h}$ with a secondary antibody [horseradish peroxidase (HRP)-conjugated goat antimouse IgG]. The chemiluminescence was detected using ECL Plus as previously described [20].

\subsection{Preparation of RNA and Quantitative Real-Time Polymerase Chain Reaction (PCR)}

Six days after RANKL stimulation (50 $\mathrm{ng} / \mathrm{ml}$ ), total RNA was extracted from RAW264.7 cells using ReliaPrep RNA Cell Miniprep System and subjected to reverse transcription. Quantitative real-time PCR was performed using StepOnePlus (Applied Biosystems, Waltham, MA, USA) under the following conditions: $30 \mathrm{sec}$ at $95^{\circ} \mathrm{C}$, followed $5 \mathrm{sec}$ at $95^{\circ} \mathrm{C}$ and $30 \mathrm{sec}$ at $60^{\circ} \mathrm{C}$ for 40 cycles. Target gene expression was normalized to glyceraldehyde 3-phosphate dehydrogenase (Gapdh) mRNA. The osteoclastassociated genes were selected based on Nakamura et al.'s report [21]. The sequences of the primers were obtained from PrimerBank and have the following IDs: Tnfrsf11a, 
31981958a1; Nfatc1, 31560459a1; Mafb, 23308601a1; Prdm1, 6680784a1; Ctsk, 31982433a1; Dcstamp, 12852706a1; Ocstamp 21312818a1; Atp6vOd2, 29150255a1; and Gapdh, 6679937a1.

\subsection{Statistical Analysis}

All results are expressed as the means with S.E.M., and the statistical significance between two groups was determined by Student's t-test or Aspin-Welch test, and with regard to multiple comparisons, statistical significance was determined by parametric Dunnett test. Data were analyzed with statistical programs (SAS release 8.2 or 9.2, SAS Institute Japan Ltd., Tokyo, Japan; Stat Preclinica 1.2, Takumi Information Technology, Tokyo, Japan). In all tests, $\mathrm{p}<0.05$ (two-tails) was considered to be significant. For in vitro variables, the independent experiments were repeated three times.

\section{Results}

\subsection{Effect of IGU on Bone Structural Integrity of the Arthritic Joints}

AIA rats showed chronic paw swelling, aggressive synovitis, cartilage degradation, and bone erosion not only in injected paws, but also in non-injected paws. In this study, the structural integrity of the hind paws was observed on the bone structure of calcaneum, on which a quantitative analysis is possible [18]. Swelling of both hind paws peaked approximately 3 weeks after the induction. On day 21, the swelling rates in the injected and non-injected paws were approximately 2.5 -fold and 2-fold higher than those in the normal group, respectively (Figure 1(a)). As shown in Figures 1(i)-(n), $\mu$ CT images in the control group indicated the development of osteoporosis in the whole calcaneal bones. BMD and bone morphometry parameters of the calcaneum in non-injected paws were calculated from each $\mu \mathrm{CT}$ image. In the IGU-administered group, paw swelling was inhibited (Figure 1(a)) and the calcaneum BMD was rescued by IGU administration (Figure $1(\mathrm{~b})$ ). The trabecular bone density was analyzed by BV/TV (Figure 1(c)) and trabecular bone structure was analyzed with TBPf (Figure 1(d)) and SMI (Figure 1(e)). IGU administration suppressed the pathological changes observed in the bone structural parameters. All images and bone morphometry parameters were obtained in the non-injected paws because the lesion induced by adjuvant injection would affect these parameters. These data demonstrated that IGU abolished severe bone resorption in AIA rats.

Bone resorption is triggered by excessive activation of osteoclasts. Therefore they were visualized with TRAP staining in the calcanea of the non-injected paws, in which obvious osteoporosis was observed (Figure 2(a) and Figure 2(b); magnified image, Figure 2(d) and Figure 2(f)). In the control group, many TRAP-positive cells were observed in the whole calcanea, not just in peri-articular sites. The number of osteoclast cells in the calcanea was less than 1/10 in the IGU-administered group (Figure 2(c)). Furthermore, hematoxylin-eosin staining was performed. While aggressive synovitis, pannus formation in the subtalar joint and accelerated trabecular bone resorption were observed in the control group (Figure 2(d) and Figure 2(e)), osteoclasts and bone ero- 

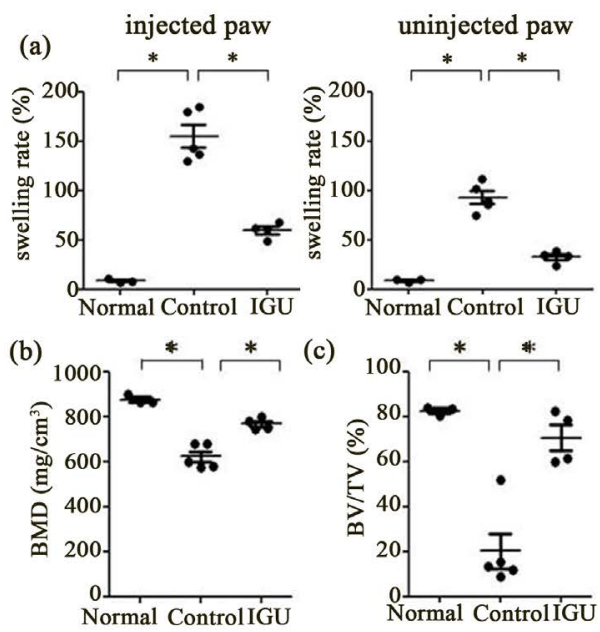

(c)
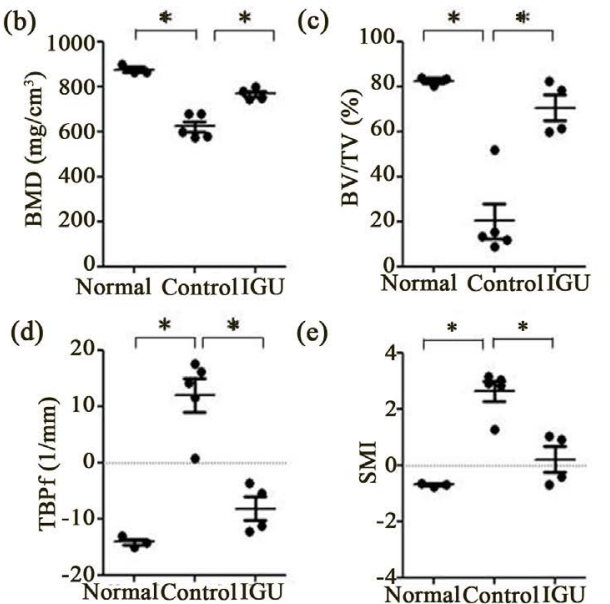

(e)
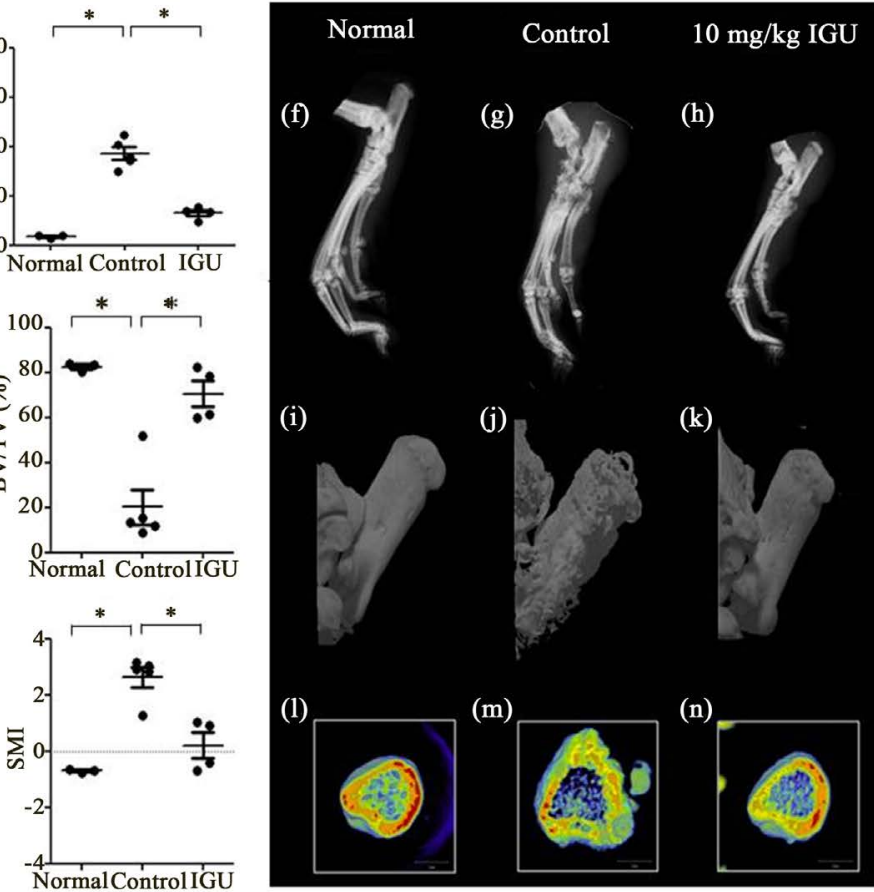

Figure 1. Effect of IGU on bone destruction in adjuvant-induced arthritis (AIA) rats. Arthritis was induced by intradermal injection of killed M. tuberculosis in the left hind paws on day 0 . IGU at $10 \mathrm{mg} / \mathrm{kg} /$ day was administered orally once daily from day 0 to day 20 . The hind paws were collected on day 21. (a) The swelling rate (\%) (b) Bone mineral density of the calcaneum. Bone morphometry parameters: (c) Bone volume per trabecular volume (BV/TV); (d) Trabecular bone pattern factor (TBPf); and (e) Structural model index (SMI). X-ray images of (f) Normal, (g) Control and (h) IGU group. Three-dimensional calcaneum images using $\mu \mathrm{CT}$ images: (i) Normal, (j) Control and (k) IGU group. Section images: (l) Normal, (m) Control and (n) IGU group. Data are expressed as individual values and means with S.E.M., $\mathrm{n}=3-5,{ }^{\star}: p<0.05$.

sion were only slightly observed at the synovial-bone interface in the IGU-administered group (Figure 2(f) and Figure 2(g)).

\subsection{Effect of IGU on Osteoclast Differentiation and Bone Resorbing Activity in RANKL-Stimulated RAW264.7 Cells}

To determine how IGU acts on osteoclast differentiation, we examined the effect of IGU on osteoclast-formation from RAW264.7 cells. After being cultured with $50 \mathrm{ng} / \mathrm{ml}$ of RANKL, RAW264.7 cells were differentiated into TRAP-positive multinucleated cells. IGU at 1 and $10 \mu \mathrm{M}$ inhibited the differentiation of RAW264.7 cells into TRAPpositive cells (Figure 3(a) and Figure 3(b)). Moreover, IGU at 0.1 to $10 \mu \mathrm{M}$ decreased TRAP activity in a concentration-dependent manner (Figure 3(c)). The cell viability was not affected with by IGU at these concentrations (data not shown). Next, in order to investigate the function of the differentiated cells, bone resorbing activity was measured in RANKL-stimulated RAW264.7 cells. Figure 3(d) shows the images of bone resorption (pit formation) on day 9 and Figure 3(e) shows bone resorption activity on 

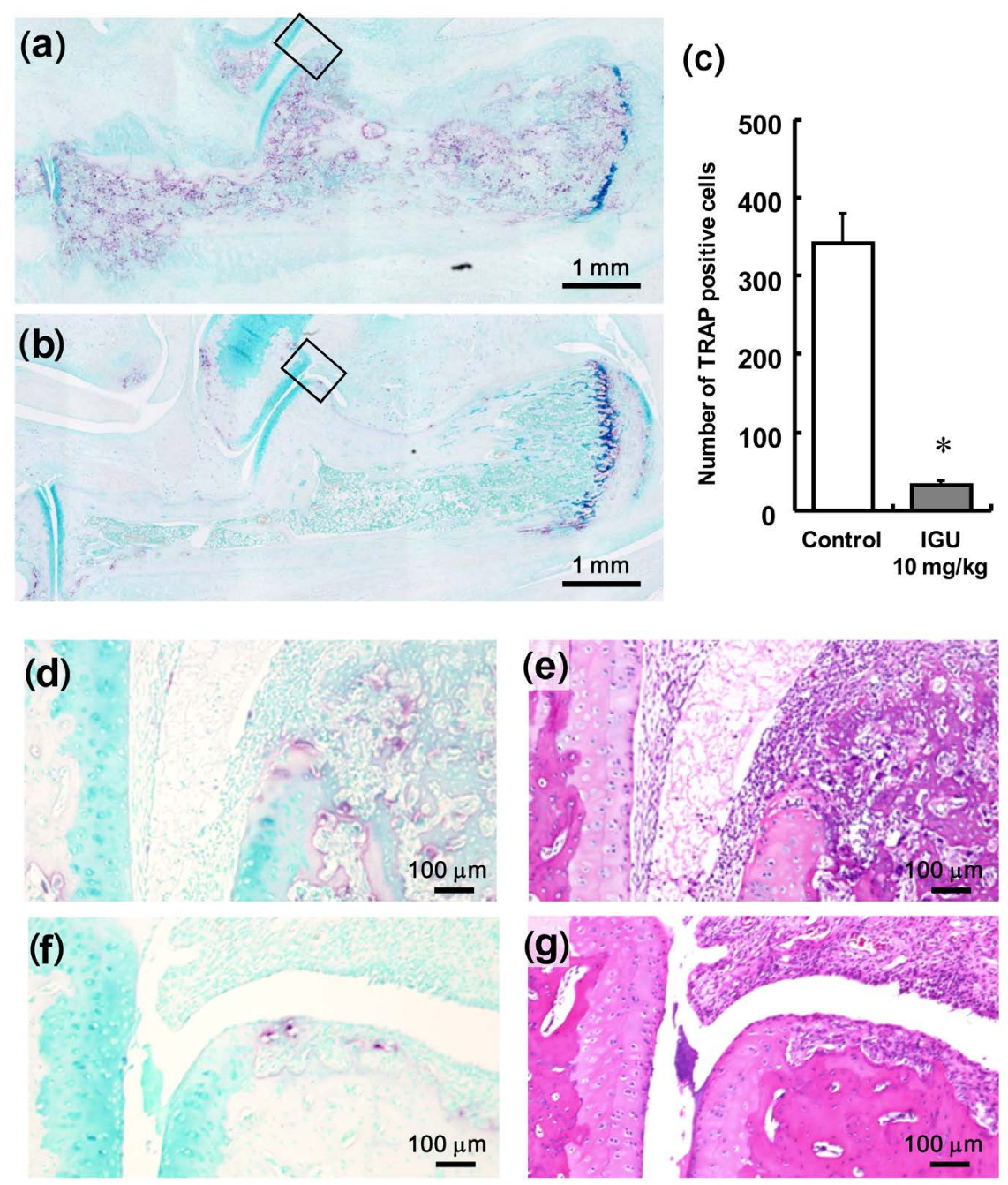

Figure 2. Histological analysis of vehicle- or IGU-treated AIA rats on day 21. ((a), (b)) TRAP staining of the calcaneum, ((d), (f)) magnified image of opened squares, ((e), (g)) Hematoxylin-eosin staining. (a), (d), and (e), control group; (b), (f), and (g), $10 \mathrm{mg} / \mathrm{kg}$ IGU-administered group. (c) Number of TRAP-positive cells in the sections of the calcaneum from the non-injected hind paws. Data are expressed as means with S.E.M., $\mathrm{n}=4,{ }^{*}: p<0.05$.

day 6. IGU suppressed the RANKL-induced differentiation into osteoclast-functional cells with bone resorbing activity.

\subsection{Effect of IGU on RANKL-Induced NFATc1 Pathway}

We focused on the NFATc1 signaling pathway because it is a master regulator of osteoclast differentiation. The NFATc1 protein level was examined by immunoblotting using nuclear and cytoplasmic extracts of RANKL-stimulated RAW264.7 cells (Figure 4). The NFATc1 protein level in cells treated with IGU at $1 \mu \mathrm{M}$ or more was lower than that in control cells. The effect of IGU on NFATc1-DNA binding was investigated by DNA-binding ELISA. However, IGU at 1 to $300 \mu \mathrm{M}$ did not affect NFATc1-DNA binding activity (data not shown). 
(a)
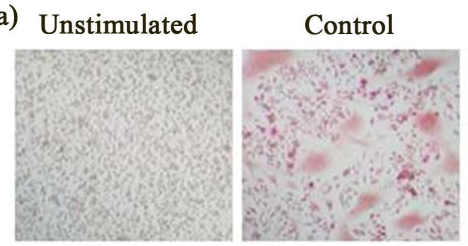

$1 \mu \mathrm{M}$ IGU

$10 \mu \mathrm{M}$ IGU

(b)

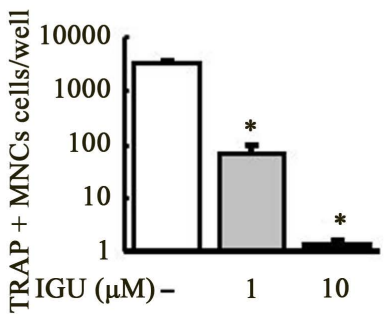

(d)

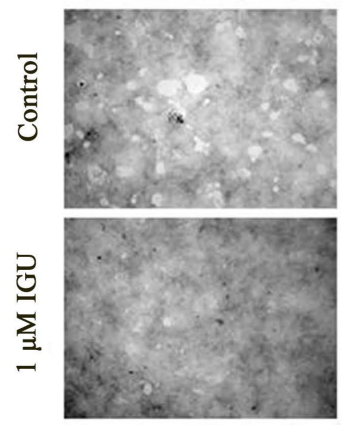

(c)

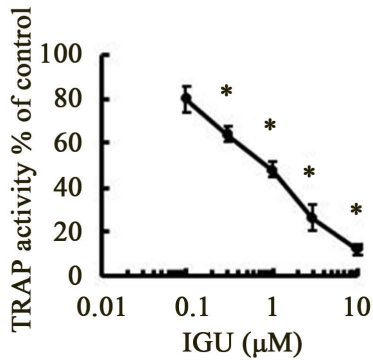

(e)

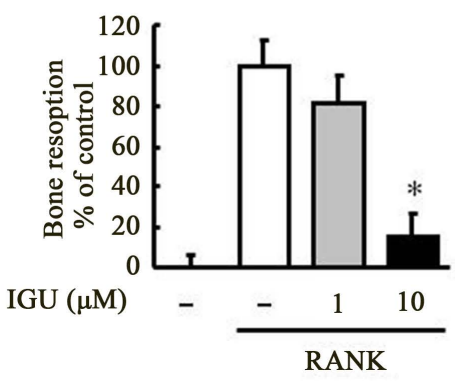

Figure 3. Effects of IGU on osteoclastogenesis in vitro. (a) Pictures of TRAP-stained cells. RAW264.7 cells were incubated in the presence of RANKL (50 ng/ml) for 6 days and cells were stained for TRAP; (b) Quantitation of TRAP-positive cells. (c) TRAP activity shown as the percentage of control; (d) Pictures of bone resorption of cells (pit formation) cultured for 9 days; (e) Fluorescence intensity of the culture supernatant on day 6. Bone resorption activity was measured with the supernatant of cells cultured on a fluoresceinated calcium phosphate-coated plate by spectrometer. Data are expressed as means with S.E.M., $\mathrm{n}=3,{ }^{*}: p<0.05$ vs. control.
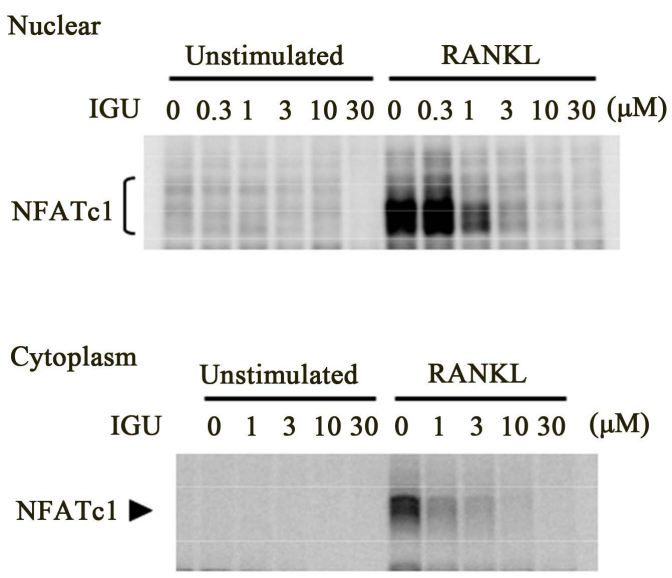

Figure 4. Expression and nuclear translocation of NFATc1 protein in RANKL-induced RAW264.7 cells. Cells were incubated in the presence of RANKL for 24 hours. Nuclear and cytoplasmic extracts (10 $\mu \mathrm{g}$ protein/lane) were prepared and immunoblotting was performed with an anti-NFATc1 mAb (7A6). 


\subsection{Effect of IGU on Osteoclast-Associated Gene Expression}

Finally, in order to investigate the detailed mechanism underlying the suppressive effect of IGU on NFATc1 protein expression and osteoclastogenesis, the expression of osteoclast-associated genes was measured in RANKL-stimulated RAW264.7 cells in the absence or presence of IGU at 0.1 to $10 \mu \mathrm{M}$ (Figure 5). We investigated the gene expression of $N f a t c 1$ and its upstream factor (tumor necrosis factor receptor superfamily, member 11a [Tnfrsf11a]). IGU inhibited Nfatc1 and Tnfrsf11a gene expression. Next, the gene expression of regulators of NFATc1 (V-maf musculoaponeurotic fibrosarcoma oncogene homolog B $[M a f b]$, and PR domain containing 1, with ZNF domain [Prdm1]) was measured. IGU normalized the change in the expression of these genes. Finally, we also investigated NFATc1-regulated osteoclast-functional genes and cell-cell fusion genes (cathepsin $\mathrm{K}$ [Ctsk], dendritic cell-specific transmembrane protein [Dcstamp], osteoclast stimulatory transmembrane protein [Ocstamp], and ATPase, H+ transporting, lysosomal $38 \mathrm{kDa}, \mathrm{V} 0$ subunit D2 [Atp6v0d2]). IGU suppressed the expression of these genes.

\section{Discussion}

In our previous studies, we demonstrated that IGU suppressed bone destructive symptoms such as osteoporosis and bone erosion in AIA rats and CIA mice [9], [17]. In this study, the 3-D bone structure of calcanea was inspected in more details using $\mu \mathrm{CT}$ analysis in AIA rats. In the control group, bone resorption was accelerated not only in the cortical bones, but also in the cancellous bones. IGU suppressed the development of such osteoporotic lesions and significantly decreased the number of osteoclasts in the

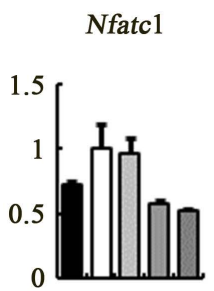

Ctsk

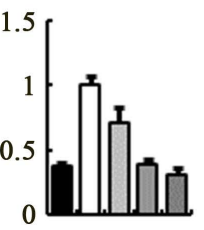

Tnfrsf11a
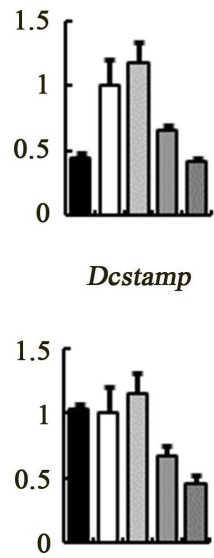

Mafb
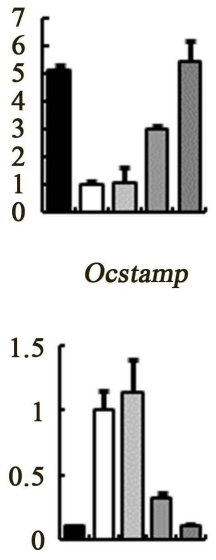

$\operatorname{Prdm} 1$

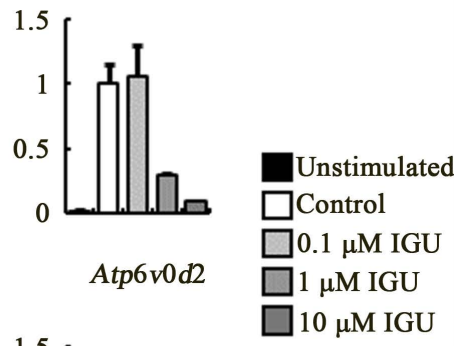

Figure 5. Effect of IGU on osteoclast-associated gene expression in RANKL-stimulated RAW264. 7 cells. Cells were incubated in the presence of RANKL for 6 days. Total RNA was extracted and mRNA levels were examined by quantitative real-time PCR. The expression of the factors upstream of NFATc1, Tnfrsf11a; the negative regulator of NFATc1, Mafb; the negative regulator of MafB, Prdm1; NFATc1-regulated osteoclast functional gene, Ctsk, and NFATc1-regulated genes required for cell-cell fusion, Dcstamp, Ocstamp and Atp6v0d2 was examined. Data are expressed as means with S.E.M., $\mathrm{n}=3$, and the results are representative of three independent experiments. 
calcanea of AIA rats. Based on these results, we assumed that IGU would inhibit osteoclast differentiation. Therefore, in vitro culture experiments were conducted using RAW264.7 cells to determine whether IGU has an inhibitory effect on osteoclast differentiation. As a result, this drug inhibited the differentiation of RANKL-stimulated RAW264.7 cells into osteoclasts, which were identified morphologically and functionally, and it was in consistency with that reported by Gan et al. [12].

The inhibition of osteoclast differentiation by IGU in AIA model was considered to be modified by its own anti-inflammatory effect. Especially, synovial inflammation including pannus formation is known to play an important role in joint destruction followed by bone resorption. Therefore, it was of great interest whether IGU inhibits osteoclast differentiation even under the situation without inflammation and this point would be a future problem. In vitro osteoclast-formation induced with other stimuli than RANKL might be worth examining. On the one hand, it has been known that IGU stimulated osteoblastic differentiation and promoted bone morphogenetic protein-2 (BMP-2) induced bone formation by stimulation of osterix expression [13]. The association in the mechanism of both actions of suppressive effect on bone resorption and the promoting effect on bone formation is unidentified, but it was again confirmed that IGU possessed anabolic action for bone metabolism.

Recently, NFATc1 was defined as a master transcription factor in osteoclast differentiation [15], [22]. NFATc1 protein level was elevated by RANKL-stimulation and reduced by IGU treatment. We next investigated the effect of IGU on the expression of $N f a t c 1$, its regulator genes, upstream factor, osteoclast-functional gene, and cell-cell fusion genes using RANKL-stimulated RAW264.7 cells. In our experimental conditions, Nfatcl expression level was not dramatically elevated, but IGU normalized its expression. Moreover, IGU amplified Mafb expression and inhibited Prdm1 gene expression. In the NFATc1-osteoclastogenesis pathway, $M a f B$ acts as a negative regulator [23], and the expression of $M a f B$ is suppressed by Blimp1 (encoded by $\operatorname{Prdml}$ ) protein [24]. Prdm1 expression is controlled by the transcription factor, NFATc1 [24]. Blimp1 is a key regulator of B cell differentiation into plasma cells [25]. Moreover, since IGU suppresses immunoglobulin production by directly acting on B cells [6], IGU may suppress not only osteoclastogenesis, but also immunoglobulin production by directly inhibiting Prdm1 expression. On the other hand, IGU also suppressed Tnfrsf11a (Rank) expression. It has been reported that Rank mRNA is enhanced by RANKL stimulation [26], therefore the mRNA expression level of Tnfrsf11a would be elevated in the control group.

Pro-inflammatory cytokines such as IL- $1 \beta$, IL-6, MCP-1, and TNF- $\alpha$ are involved in RANKL-induced osteoclastogenesis [27] [28] [29] [30]. We and Kohno et al. [5], [31], [32] demonstrated that IGU suppresses the production of these cytokines through inhibiting NF- $\mathrm{kB}$ activation in cultured monocytes/macrophages and human synoviocytes. From these previous results and this study, IGU would suppress osteoclastogenesis through pleiotropic mechanisms, i.e., IGU inhibits Rank/RANKL expression by suppressing the expression of pro-inflammatory cytokines and suppresses the expres- 
sion of the transcription factor NFATc1 without affecting RANKL expression in osteoclast precursor cells. The concentrations at which IGU suppresses osteoclastogenesis in AIA rats and RANKL-stimulated RAW264.7 cells are similar to the plasma levels observed in patients with RA [5]. Therefore, IGU should inhibit bone destruction in RA via suppressing bone resorption and osteoclastogenesis when used in the clinic.

\section{Conclusion}

In conclusion, we showed that IGU suppressed the bone resorption and lesions of bone trabeculae with accompanying a significant decrease of osteoclasts in the calcaneal bones of AIA rats. Moreover, this drug inhibited the in vitro differentiation of RANKL-stimulated RAW264.7 cells into osteoclasts. IGU decreased the amount of NFATc1 protein and improved the altered expression of NFATc1-associated genes and osteoclast-functional genes. Therefore, it was found that IGU suppressed osteoclastogenesis and bone resorption via the RANKL-NFATc1 pathway, suggesting such effect would be expected in clinical use.

\section{Acknowledgements}

Not applicable.

\section{Declarations}

Ethics approval and consent to participate: The animal studies were conducted in accordance with the laboratory animal use management regulation at Toyama Chemical, based on "Principles of Laboratory Animal Care".

\section{Competing Interests}

All authors are employees of Toyama Chemical Co., Ltd., the manufacturer of iguratimod.

\section{Authors' Contributions}

J.S. and H.M. contributed equally to this work. All authors read and approved the final manuscript.

\section{References}

[1] Tanaka, K., Yamaguchi, T. and Hara, M. (2015) Iguratimod for the Treatment of Rheumatoid Arthritis in Japan. Expert Review of Clinical Immunology, 11, 565-573. http://dx.doi.org/10.1586/1744666X.2015.1027151

[2] Hara, M., Abe, T., Sugawara, S., Mizushima, Y., Hoshi, K., Irimajiri, S., et al. (2007) Efficacy and Safety of Iguratimod Compared with Placebo and Salazosulfapyridine in Active Rheumatoid Arthritis: A Controlled, Multicenter, Double-Blind, Parallel-Group Study. Modern Rheumatology, 17, 1-9. http://dx.doi.org/10.3109/s10165-006-0542-y

[3] Ishiguro, N., Yamamoto, K., Katayama, K., Kondo, M., Sumida, T., Mimori, T., et al. (2013) Concomitant Iguratimod Therapy in Patients with Active Rheumatoid Arthritis despite Stable Doses of Methotrexate: A Randomized, Double-Blind, Placebo-Controlled Trial. 
Modern Rheumatology, 23, 430-439. http://dx.doi.org/10.3109/s10165-012-0724-8

[4] Okamura, K., Yonemoto, Y., Okura, C., Kobayashi, T. and Takagishi, K. (2015) Efficacy of the Clinical Use of Iguratimod Therapy in Patients with Rheumatoid Arthritis. Modern Rheumatology, 25, 235-240. http://dx.doi.org/10.3109/14397595.2014.938401

[5] Tanaka, K. (2009) Iguratimod (T-614): A Novel Disease Modifying anti-Rheumatic Drug. Rheumatology Reports, 1, e4. http://dx.doi.org/10.4081/rr.2009.e4

[6] Tanaka, K., Yamamoto, T., Aikawa, Y., Kizawa, K., Muramoto, K., Matsuno, H., et al. (2003) Inhibitory Effects of an Anti-Rheumatic Agent T-614 on Immunoglobulin Production by Cultured B Cells and Rheumatoid Synovial Tissues Engrafted into SCID Mice. Rheumatology (Oxford), 42, 1365-1371. http://dx.doi.org/10.1093/rheumatology/keg381

[7] Inaba, T., Tanaka, K., Takeno, R., Nagaki, H., Yoshida, C. and Takano, S. (200) Synthesis and Antiinflammatory Activity of 7-Methanesulfonylamino-6-Phenoxychromones. Antiarthritic Effect of the 3-Formylamino Compound (T-614) in Chronic Inflammatory Disease Models. Chemical and Pharmaceutical Bulletin (Tokyo), 48, 131-139.

http://dx.doi.org/10.1248/cpb.48.131

[8] Aikawa, Y., Tanuma, N., Shin, T., Makino, S., Tanaka, K. and Matsumoto, Y. (1988) A New Anti-Rheumatic Drug, T-614, Effectively Suppresses the Development of Autoimmune Encephalomyelitis. Journal of Neuroimmunology, 89, 35-42.

http://dx.doi.org/10.1016/S0165-5728(98)00056-3

[9] Tanaka, K., Shimotori, T., Taniguchi, Y., Eguchi, M. and Abe, C. (1993) Pharmacological Studies on T-614, a Novel Antiinflammatory Agent: Effect on Type II Collagen-Induced Arthritis in DBA/1J Mice and Spontaneous Arthritis in MRL/l Mice. Int J Immunotherapy, IX, 69-78.

[10] Luo, Q., Sun, Y., Liu, W., Qian, C., Jin, B., Tao, F., et al. (2013) A Novel Disease-Modifying Antirheumatic Drug, Iguratimod, Ameliorates Murine Arthritis by Blocking IL-17 Signaling, distinct From Methotrexate and Leflunomide. Journal of Immunology, 191, 4969-4978. http://dx.doi.org/10.4049/jimmunol.1300832

[11] Du, F., Lü, L.J., Fu, Q., Dai, M., Teng, J.L., Fan, W., et al. (2008) T-614, a Novel Immunomodulator, Attenuates Joint Inflammation and Articular Damage in Collagen-Induced Arthritis. Arthritis Research \& Therapy, 10, R136. http://dx.doi.org/10.1186/ar2554

[12] Gan, K., Yang, L., Xu, L., Feng, X., Zhang, Q., Wang, F., et al. (2014) Iguratimod (T-614) Suppresses RANKL-Induced Osteoclast Differentiation and Migration in RAW264.7 Cells via NF- $\mathrm{BB}$ and MAPK Pathways. International Immunopharmacology, 35, 294-300. http://dx.doi.org/10.1016/j.intimp.2016.03.038

[13] Kuriyama, K., Higuchi, C., Tanaka, K., Yoshikawa, H. and Itoh, K. (2002) A Novel Anti-Rheumatic Drug, T-614, Stimulates Osteoblastic Differentiation in Vitro and Bone Morphogenetic Protein-2-Induced Bone Formation in Vivo. Biochemical and Biophysical Research Communications, 299, 903-909. http://dx.doi.org/10.1016/S0006-291X(02)02754-7

[14] Takayanagi, H., Kim, S., Koga, T., Nishina, H., Isshiki, M., Yoshida, H., et al. (2002) Induction and Activation of the Transcription Factor NFATc1 (NFAT2) Integrate RANKL Signaling in Terminal Differentiation of Osteoclasts. Developmental Cell, 3, 889-901. http://dx.doi.org/10.1016/S1534-5807(02)00369-6

[15] Urushibara, M., Takayanagi, H., Koga, T., Kim, S., Isobe, M., Morishita, Y., et al. (2004) The Antirheumatic Drug Leflunomide Inhibits Osteoclastogenesis by Interfering with Receptor Activator of NF-Kappa B Ligand-Stimulated Induction of Nuclear Factor of Activated T Cells c1. Rheumatoid Arthritis, 50, 794-804. http://dx.doi.org/10.1002/art.20206

[16] Asagiri, M., Sato, K., Usami, T., Ochi, S., Nishina, H., Yoshida, H., et al. (2005) Autoamplification of NFATc1 Expression Determines Its Essential Role in Bone Homeostasis. Journal 
of Experimental Medicine, 202, 1261-1269. http://dx.doi.org/10.1084/jem.20051150

[17] Tanaka, K., Makino, S., Shimotori, T., Aikawa, Y., Inaba, T. and Yoshida, C. (1992) Pharmacological Studies of the New Antiinflammatory Agent 3-Formylamino-7-Methylsulfonylamino-6-Phenoxy-4H-1-Benzopyran-4-One. 1st Communication: Antiinflammatory, Analgesic and Other Related Properties. Arzneimittelforschung, 42, 935-944.

[18] Noguchi, M., Kimoto, A., Sasamata, M. and Miyata, K. (2008) Micro-CT Imaging Analysis for the Effect of Celecoxib, a Cyclooxygenase-2 Inhibitor, on Inflammatory Bone Destruction in Adjuvant Arthritis Rats. Journal of Bone and Mineral Metabolism, 26, 461-468. http://dx.doi.org/10.1007/s00774-008-0855-3

[19] Hirotani, H., Tuohy, N.A., Woo, J.T., Stern, P.H. and Clipstone, N.A. (2004) The Calcineurin/Nuclear Factor of Activated T Cells Signaling Pathway Regulates Osteoclastogenesis in RAW264.7 Cells. Journal of Biological Chemistry, 279, 13984-13992. http://dx.doi.org/10.1074/jbc.M213067200

[20] Aikawa, Y., Morimoto, K., Yamamoto, Y., Chaki, H., Hashiramoto, A., Narita, H., et al. (2008) Treatment of Arthritis with a Selective Inhibitor of $c$-Fos/Activator Protein-1. Nature Biotechnology, 26, 817-823. http://dx.doi.org/10.1038/nbt1412

[21] Nakamura, H., Nakashima, T., Hayashi, M., Izawa, N., Yasui, T., Aburatani, H., et al. (2014) Global Epigenomic Analysis Indicates Protocadherin-7 Activates Osteoclastogenesis by Promoting Cell-Cell Fusion. Biochemical and Biophysical Research Communications, 455, 305-311. http://dx.doi.org/10.1016/j.bbrc.2014.11.009

[22] Nakashima, T., Hayashi, M. and Takayanagi, H. (2012) New Insights into Osteoclastogenic Signaling Mechanisms. Trends in Endocrinology and Metabolism, 23, 582-590. http://dx.doi.org/10.1016/j.tem.2012.05.005

[23] Kim, K., Kim, J.H., Lee, J., Jin, H.M., Kook, H., Kim, K.K., et al. (2007) MafB Negatively Regulates RANKL-Mediated Osteoclast Differentiation. Blood, 109, 3253-3259. http://dx.doi.org/10.1182/blood-2006-09-048249

[24] Nishikawa, K., Nakashima, T., Hayashi, M., Fukunaga, T., Kato, S., Kodama, T., et al. (2010) Blimp1-Mediated Repression of Negative Regulators Is Required for Osteoclast Differentiation. Proceedings of the National Academy of Sciences of the United States of America, 107, 3117-3122. http://dx.doi.org/10.1073/pnas.0912779107

[25] Shapiro-Shelef, M., Lin, K.I., McHeyzer-Williams, L.J., Liao, J., McHeyzer-Williams, M.G. and Calame, K. (2003) Blimp-1 Is Required for the Formation of Immunoglobulin Secreting Plasma Cells and Pre-Plasma Memory B Cells. Immunity, 19, 607-620. http://dx.doi.org/10.1016/S1074-7613(03)00267-X

[26] Kido, S., Inoue, D., Hiura, K., Javier, W., Ito, Y. and Matsumoto, T. (2003) Expression of RANK Is Dependent upon Differentiation into the Macrophage/Osteoclast Lineage: Induction by 1Alpha,25-Dihydroxyvitamin $\mathrm{D}_{3}$ and TPA in a Human Myelomonocytic Cell Line, HL60. Bone, 32, 621-629. http://dx.doi.org/10.1016/S8756-3282(03)00049-8

[27] Wei, S., Kitaura, H., Zhou, P., Ross, F.P. and Teitelbaum, S.L. (2005) IL-1 Mediates TNF-Induced Osteoclastogenesis. Journal of Clinical Investigation, 115, 282-290. http://dx.doi.org/10.1172/JCI200523394

[28] Wong, P.K., Quinn, J.M., Sims, N.A., van Nieuwenhuijze, A., Campbell, I.K. and Wicks, I.P. (2006) Interleukin-6 Modulates Production of T lymphocyte-Derived Cytokines in Antigen-Induced Arthritis and Drives Inflammation-Induced Osteoclastogenesis. Arthritis \& Rheumatology, 54, 158-168. http://dx.doi.org/10.1002/art.21537

[29] Miyamoto, K., Ninomiya, K., Sonoda, K.H., Miyauchi, Y., Hoshi, H., Iwasaki, R., et al. (2009) MCP-1 Expressed by Osteoclasts Stimulates Osteoclastogenesis in an Autocrine/ 
Paracrine Manner. Biochemical and Biophysical Research Communications, 383, 373-377. http://dx.doi.org/10.1016/j.bbrc.2009.04.020

[30] Nakao, A., Fukushima, H., Kajiya, H., Ozeki, S. and Okabe, K. (2007) RANKL-Stimulated TNF Alpha Production in Osteoclast Precursor Cells Promotes Osteoclastogenesis by Modulating RANK Signaling Pathways. Biochemical and Biophysical Research Communications, 357, 45-50. http://dx.doi.org/10.1016/j.bbrc.2007.04.058

[31] Aikawa, Y., Yamamoto, M., Yamamoto, T., Morimoto, K. and Tanaka, K. (2002) An AntiRheumatic Agent T-614 Inhibits NF-kB Activation in LPS- and TNF- $\alpha$-Stimulated THP-1 Cells without Interfering with IкB $\alpha$ Degradation. Inflammation Research, 51, 188-194. http://dx.doi.org/10.1007/PL00000291

[32] Kohno, M., Aikawa, Y., Tsubouchi, Y., Hashiramoto, A., Yamada, R., Kawahito, Y., et al. (2001) Inhibitory Effect of T-614 on Tumor Necrosis Factor- $\alpha$ Induced Cytokine Production and Nuclear Factor- $\kappa \mathrm{B}$ Activation in Cultured Human Synovial Cells. Journal of Rheumatology, 28, 2591-2596.

\section{List of Abbreviations}

IGU: iguratimod, AIA: adjuvant-induced arthritis, RANKL: receptor activator of nuclear factor kappa-B ligand, $\mu \mathrm{CT}$ : micro computed tomography, TRAP: tartrate-resistant acid phosphatase, NFATc1: nuclear factor of activated T-cells, cytoplasmic, calcineurin-dependent 1, DMARD: disease-modifying anti-rheumatic drug, RA: rheumatoid arthritis, NF-kB: nuclear factor kappa-B, CIA: collagen-induced arthritis, MAPK: mitogen-activated protein kinase, DMEM: Dulbecco's modified Eagle medium, FBS: fetal bovine serum, NEAA: non-essential amino acid, mAb: monoclonal antibody, BMD: bone mineral density, BV/TV: bone volume/tissue volume, TBPf: trabecular bone pattern factor, SMI: structural model index, ALP: alkaline phosphatase, MNC: multinucleated cell, TBS: Tris buffered saline, HRP: horseradish peroxidase, PCR: polymerase chain reaction, BMP-2: bone morphogenetic protein-2, IL-1 $\beta$ : interleukin- $1 \beta$, IL-6: interleukin-6, MCP-1: monocyte chemotactic protein-1, TNF- $\alpha$ : tumor necrosis factor- $\alpha$. 